\title{
Solvent Effects in Acid-Catalyzed Dehydration of the Diels-Alder Cycloadduct between 2,5-Dimethylfuran and Maleic Anhydride
}

\author{
Taha Salavati-fard ${ }^{1,2^{*}}$, Stavros Caratzoulas ${ }^{2}$ and Douglas J Doren ${ }^{2,3}$
}

${ }^{1}$ Department of Physics and Astronomy, University of Delaware, Newark, Delaware 19716

${ }^{2}$ Catalysis Center for Energy Innovation (CCEI), Department of Chemical and Biomolecular Engineering, University of Delaware, Newark, Delaware 19716

${ }^{3}$ Department of Chemistry and Biochemistry, University of Delaware, Newark, Delaware 19716

Keywords: Dehydration; Diels-Alder cycloadduct; Solvent effects; Dimethylfuran; DFT.

\begin{abstract}
Dehydration of the cycloadduct produced from the Diels-Alder reaction between 2,5dimethylfuran and maleic anhydride to 3,6-dimethylphthalic anhydride exemplifies an important step in producing platform chemicals from biomass. The mechanisms of dehydration and catalytic effects of Lewis and Brønsted acids are investigated with density functional theory. The uncatalyzed reaction has a very high activation barrier $(68.7 \mathrm{kcal} / \mathrm{mol})$ in the gas phase and it is not significantly affected by solvation. With a Lewis acid catalyst, modeled as an alkali ion, the activation barriers are reduced, but intermediates are also stabilized. The net effect in vacuum is
\end{abstract}


that the energetic span, or apparent activation energy of the catalytic cycle, is $77.9 \mathrm{kcal} / \mathrm{mol}$, even higher than the barrier in the uncatalyzed case. In solution, however, the energetic span is reduced by as much as $20 \mathrm{kcal} / \mathrm{mol}$, due to differences in the solvation energy of the transition states and intermediates. In the case of a Brønsted acid catalyst, modeled as a proton, the gas phase transition state energies are reduced even more than in the Lewis acid case, and there is no strong stabilization of the intermediates. The energetic span in vacuum is only $13.8 \mathrm{kcal} / \mathrm{mol}$ and is reduced even further in solution. Brønsted acid catalysis appears to be the preferred mechanism for dehydration of this cycloadduct. Since the Diels-Alder reaction that produced the molecule has previously been shown to be catalyzed by Brønsted acids, this suggests that a single catalyst could be used to accelerate both steps.

\section{Introduction}

Biomass conversion to fuels and chemicals has opened a promising arena for sustainable energy generation and greenhouse gas emissions reduction. ${ }^{1-6}$ Producing aromatic molecules from biomass-derived chemicals is an important step toward making use of biomass in the chemical industry. One such path uses an approach developed by Brion, using Diels-Alder cycloaddition to a furan, followed by cycloadduct dehydration. ${ }^{7}$ An example is shown in Scheme 1, using 2,5-

dimethylfuran (DMF), which is readily derived from biomass. ${ }^{8-9}$ Here, the cycloaddition of maleic anhydride (MA) and DMF produces an oxa-norbornene derivative, which can dehydrate to the aromatic product (3,6-dimethylphthalic anhydride). The latter reaction is the focus of this paper. This aromatic product is an important molecule used in the manufacture of plasticizers, unsaturated polyesters and alkyd resins. 
Scheme 1. Diels-Alder cycloaddition followed by dehydrative aromatization.

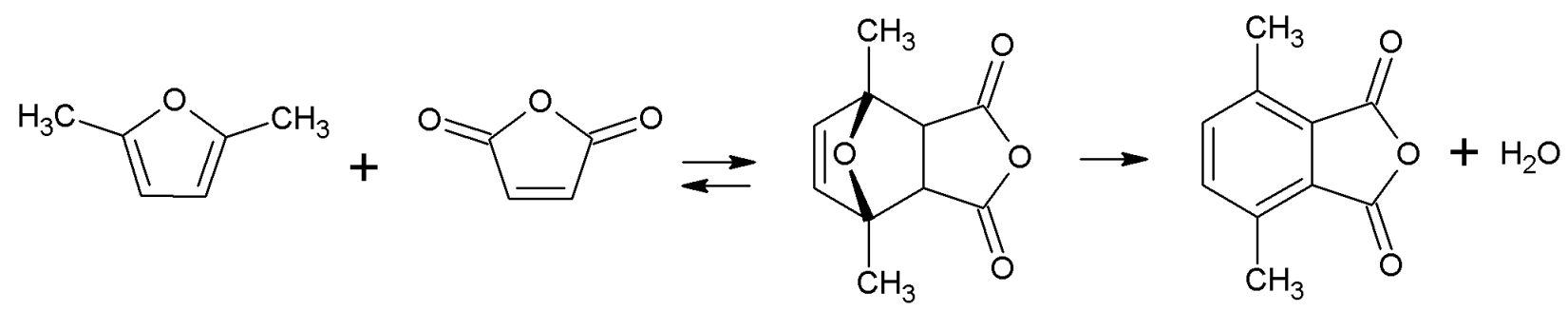

Mahmoud et al. have reported experimental studies of the production of phthalic anhydride from biomass-derived furan and MA through Diels-Alder cycloaddition followed by dehydration in the condensed-phase. ${ }^{10}$ They found excellent yields for the Diels-Alder reaction and very high selectivity to phthalic anhydride and phthalic acid for the dehydration. In related work, Nikbin et al. used computational methods to explore the effect of Lewis and Brønsted acids on the cycloaddition between DMF and ethylene, and on the subsequent dehydration of the cycloadduct to form p-xylene. ${ }^{11}$ Their gas-phase calculations showed that the dehydration reaction barrier is too high to take place without a catalyst. They found that a strong Lewis acid, such as $\mathrm{Li}^{+}$, or a Brønsted acid are able to lower the activation barrier of dehydration. ${ }^{11}$ Nikbin and coworkers have also studied the conversion with zeolite acid catalysts. ${ }^{12}$

As a means of engineering reactions for biomass conversion, solvent effects have recently attracted considerable attention on account of their impact on reaction kinetics and product distribution. ${ }^{13-16}$ We have recently reported studies on solvent effects in the Diels-Alder reaction between DMF and MA, with and without a Lewis or Brønsted acid. ${ }^{17}$ In the gas phase, Lewis acids catalyze the reaction by closing the FMO gap ( $\left.\mathrm{LUMO}_{\mathrm{MA}}-\mathrm{HOMO}_{\mathrm{DMF}}\right)$, while a Brønsted acid changes the reaction mechanism from concerted to step-wise and eliminates the activation free energy. Using a continuum solvation model, we showed that the dipole moment of the 
transition state strongly influenced the activation barrier for the acid-catalyzed cycloaddition, while non-electrostatic solvent effects also played an important role. ${ }^{17}$

In this study, using density functional theory, we investigate mechanisms for dehydration of the cycloadduct produced from the Diels-Alder reaction between DMF and MA to produce 3,6dimethyl phthalic anhydride. We consider uncatalyzed, Lewis acid catalyzed, and Brønsted acid catalyzed mechanisms, in the gas-phase and in solvent. In the next section, we describe the computational methods used. We then discuss mechanisms for uncatalyzed and acid-catalyzed dehydration in the gas-phase and follow up with a description of solvent effects. We conclude with some comments on related studies and the most promising mechanisms for application.

\section{Computational Methods and Models}

All calculations were performed with the Gaussian09 suite of programs ${ }^{18}$ using the restricted and unrestricted M06-2X hybrid density functional method ${ }^{19-20}$ with the $6-311+G(d, p)$ basis set. All structures were fully relaxed. Vibrational frequency calculations confirmed the character of minima and first-order saddle points on the potential energy hypersurface. Intrinsic reaction coordinate calculations were also performed for all transition states. Gibbs free energy levels were calculated at standard temperature and pressure i. e. $\mathrm{T}=298.15 \mathrm{~K}$ and $\mathrm{P}=100 \mathrm{kPa}$. Solvent effects have been modeled with SMD. ${ }^{21}$ To calculate partial charges on atoms, we utilize the natural bond orbital (NBO 3.1 program) analysis. ${ }^{22-24}$ In the present study, we have used simple models for Lewis and Brønsted acids (alkali ion and proton) which do not consider the zeolite environment. In light of recently published studies by Nikbin et al. ${ }^{11}$ and also by Wannakao et al. $^{25}$, we expect this to provide a qualitative understanding of the problem. 
We should also note that even though Molecular Dynamics simulations of aqueous $\mathrm{NaCl}$ solutions show the formation of contact ion pairs, at low to moderate concentrations (up to $4.5 \mathrm{M}$ ) both the $\mathrm{Na}^{+}$and $\mathrm{Cl}^{-}$ions are mostly hydrated. The hydration sphere of $\mathrm{Na}^{+}$consists of ca. 6 water molecules and that of $\mathrm{Cl}^{-}$of ca. 7 water molecules. At these concentrations, the calculated radial distribution functions show that the hydrated $\mathrm{Na}^{+}$and $\mathrm{Cl}^{-}$ions are separated by about 5.5-6 $\AA{ }^{26}$ For this reason, in the calculations presented below, we have not included counterions as that would require the consideration of a significant number of explicit water molecules in order to keep the ions from forming a concact pair.

\section{Results and discussion}

\subsection{Gas-phase}

Our calculations show that the dehydration of the cycloadduct cannot proceed uncatalyzed (Table 1). The first step is cleavage of the $\mathrm{C}_{1}-\mathrm{O}_{1}$ bond (Figs. 1 and $2 \mathrm{a}$ ), which has a barrier of $60.2 \mathrm{kcal} / \mathrm{mol}$. In the intermediate after the first transition state, $\mathrm{O}_{1}$ bridges $\mathrm{C}_{3}$ and $\mathrm{C}_{4}$ (Fig. 2a). The next step is a proton $\left(\mathrm{H}_{1}\right)$ transfer from $\mathrm{C}_{6}$ to $\mathrm{O}_{1}$ together with $\mathrm{C}_{3}-\mathrm{O}_{1}$ bond cleavage. It, too, has a high barrier, $61.1 \mathrm{kcal} / \mathrm{mol}$. We identify this step as a proton transfer because the transition state $\mathrm{NBO}$ charges of $\mathrm{H}_{1}, \mathrm{O}_{1}$ and $\mathrm{C}_{6}$ are $+0.52,-0.61$ and -0.54 , respectively. Finally, the third step of the dehydration mechanism is another proton $\left(\mathrm{H}_{2}\right)$ transfer from $\mathrm{C}_{5}$ to $\mathrm{O}_{1}$ with concerted elimination of water, crossing a barrier of $41.7 \mathrm{kcal} / \mathrm{mol}$ (Fig. 2a). The NBO charges on $\mathrm{H}_{2}, \mathrm{O}_{1}$ and $\mathrm{C}_{5}$ in the transition stare are $+0.50,-0.78$ and -0.48 , respectively, confirming the proton-like nature of the migrating hydrogen atom. 
Table 1. Gibbs free energy levels of uncatalyzed and Lewis acid catalyzed dehydration reaction in the gas-phase for alkali ions as models of Lewis acids, along with the energetic span, $\delta E$, in units of kcal/mol. Levels are indexed as in Fig. 3.

\begin{tabular}{|c|c|c|c|c|c|c|c|c|c|c|}
\hline Index & 1 & 2 & 3 & 4 & 5 & 6 & 7 & 8 & 9 & $\boldsymbol{\delta E}$ \\
\hline $\mathbf{L i}^{+}$ & 0.0 & -28.5 & 0.9 & -22.8 & 19.4 & -56.2 & -9.9 & -97.4 & -38.3 & 78.4 \\
\hline $\mathbf{N a}^{+}$ & 0.0 & -16.6 & 19.8 & -10.3 & 36.9 & -38.9 & 9.0 & -79.3 & -38.3 & 77.9 \\
\hline $\mathbf{K}^{+}$ & 0.0 & -8.3 & 30.8 & -3.4 & 47.3 & -28.3 & 10.9 & -66.5 & -38.3 & 75.5 \\
\hline None & 0.0 & 0.0 & 60.2 & 7.6 & 68.7 & -4.2 & 37.6 & -35.2 & -38.3 & --- \\
\hline
\end{tabular}

The mechanism for the Lewis acid catalyzed dehydration is shown in Fig. $2 \mathrm{~b}$ with a $\mathrm{Na}^{+}$ion used to model the acid. The mechanism is similar to that in the uncatalyzed case, but the activation barriers are substantially lower (Fig. 3 and Table 1). NBO analysis shows that our alkali ions used as model Lewis acids are not involved in any formal bonds during the dehydration, but they modify the electron density of $\mathrm{O}_{1}$ and affect the strength of proximal bonds. To analyze the impact of the ions on bond strengths, Table 2 presents bond lengths and Bader analysis ${ }^{27-29}$ for the initial structure and the two intermediates of Figs. $2 \mathrm{a}$ and $2 \mathrm{~b}$. We have considered critical points of the electron density along the $\mathrm{C}_{1}-\mathrm{O}_{1}$ bond for the initial reactant, the $\mathrm{C}_{3}-\mathrm{O}_{1}$ and $\mathrm{C}_{6}-\mathrm{H}_{1}$ bonds for the first intermediate, and the $\mathrm{C}_{5}-\mathrm{H}_{2}$ and $\mathrm{C}_{4}-\mathrm{O}_{1}$ bonds for the second intermediate. The bond critical points $(\mathrm{BCP})$, where the gradient of electron density is zero, $\nabla \rho(\boldsymbol{r})=0$, are of type $(3,-1)$ which means that two of the three eigenvalues of the Hessian matrix of $\rho$ are negative. Specifically, the electron density along a bond path has a minimum at the $\mathrm{BCP} .{ }^{29-30}$ Previous work has shown that the negative of the Laplacian of electron density, $L(\rho(\boldsymbol{r}))=-\nabla^{2}(\rho(\boldsymbol{r}))$ at the BCP is correlated with the strength of chemical bonds. ${ }^{30}$ It has also been shown that covalent bond strength increases with increasing electron density at the $\mathrm{BCP} .{ }^{31}$ 
In the cycloadduct, the first intermediate, and the second intermediate, the electron density and its Laplacian at the $\mathrm{BCP}$ along the bonds being broken $\left(\mathrm{C}_{1}-\mathrm{O}_{1}, \mathrm{C}_{3}-\mathrm{O}_{1}\right.$ and $\mathrm{C}_{4}-\mathrm{O}_{1}$, respectively) are reduced in the Lewis acid catalyzed case, relative to the uncatalyzed case (see Table 2). This indicates that these chemical bonds are stronger in the uncatalyzed case and partly accounts for the smaller energy change from the local minima to the subsequent transition state when a Lewis acid is added. The longer bond lengths when the Lewis acid is present also support the interpretation that these specific bonds are weakened by the ion. For the unreactive $\mathrm{C}_{6}-\mathrm{H}_{1}$ and $\mathrm{C}_{5}-\mathrm{H}_{2}$ bonds in the first and second intermediates, respectively, there are only small differences in the density and its Laplacian at the BCPs of the catalyzed and uncatalyzed cases, indicating little effect of the catalyst on these bonds.

Table 2. Bader analysis of the stable states that precede transition states of the uncatalyzed and Lewis acid catalyzed dehydration reactions. $\rho\left(\mathrm{e} / \AA^{3}\right)$ is electron density at the bond critical point, $L(\rho)=-\nabla^{2}(\rho)\left(\mathrm{e} / \AA^{5}\right)$ and $\mathrm{r}(\AA)$ is the bond length.

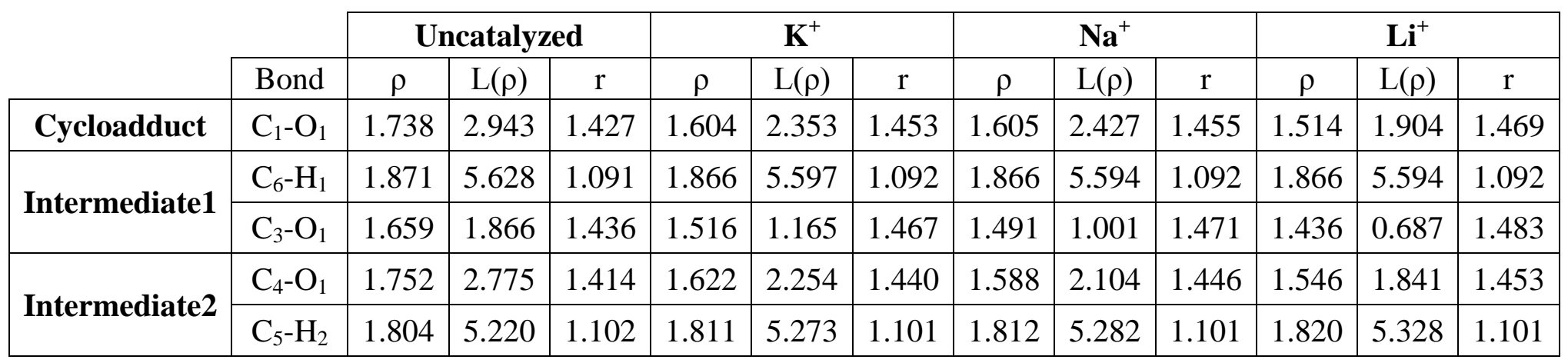

Although the activation barriers with a Lewis acid present are much lower than in the uncatalyzed case, the intermediates on the path are also stabilized.In particular, the state with the 
dehydrated product bound to the catalyst (state 8 in Fig. 3 and Table 1) is $41 \mathrm{kcal} / \mathrm{mol}$ more stable than the separated product and catalyst. As Kozuch and Shaik have pointed out, the turnover frequency for a complete catalytic cycle is determined not by the highest activation barrier, but by the energetic span of the reaction profile, the difference between the turnoverdetermining transition state (TDTS) and the turnover-determining intermediate (TDI). ${ }^{32-35}$ For the Lewis acid catalyzed dehydration, the energetic span is

$$
\delta E=\Delta G(5)-\Delta G(8)+\Delta G_{r}
$$

where $\Delta G(i)$ is the Gibbs free energy of state $i$ and $\Delta G_{r}$ is the overall free energy of reaction. Note that $\Delta G_{r}=\Delta G(9)$ in the present example. The energetic span in the case of $\mathrm{Na}^{+}$is actually higher than the activation barrier of the uncatalyzed case (Table 1). This reflects the fact that in a catalytic cycle, the largest energy change is from state 8 , with the dehydrated product bound to the catalyst, to state 5 , the highest transition state in the following dehydration. Similar results are found for $\mathrm{Li}^{+}$and $\mathrm{K}^{+}$, with shifts in activation barriers and intermediate energies compensating, so that there is little variation in the energetic span (Table 1). Thus, in the gas phase, Lewis acid catalysis is ineffective even with strong acid, at least within this simple model of the catalyst, due to the very strong binding of the product to the cation.

The mechanism of dehydration in the presence of a Brønsted acid is different from the previous cases: once the cycloadduct is protonated, only two steps are required (Fig. 2c). The first step is $\mathrm{C}_{1}-\mathrm{O}_{1}$ bond cleavage. $\mathrm{NBO}$ charges on $\mathrm{C}_{1}$ and $\mathrm{O}_{1}$ at the first transition state, are +0.47 and -0.67 . The second step is a proton transfer $\left(\mathrm{H}_{1}\right)$ from $\mathrm{C}_{6}$ to $\mathrm{O}_{1}$ and elimination of water; at the second transition state, natural charges on $\mathrm{C}_{6}, \mathrm{O}_{1}$ and $\mathrm{H}_{1}$ are $-0.42,-0.73$ and +0.52 , respectively. The activation energies between intermediates, shown for this model in Fig. 3 and Table 5, are lower 
than in the uncatalyzed or Lewis acid catalyzed cases, and there are no strongly bound intermediates. However, the reference energy is not comparable to those of the previous reactions, since it corresponds to the state with the proton bound to the cycloadduct (state 2 in Fig. 3). The final state shown is the protonated product (state $7 *$ ). However, these energies are sufficient to estimate the energetic span, since we have the relative energies of all transition states and intermediates and can calculate the overall reaction energy independently (this assumes that there is no transition state between protonated and unprotonated species, or at least no barrier high enough to change the energetic span). The energetic span in vacuum is only 13.8 $\mathrm{kcal} / \mathrm{mol}$. Thus, Brønsted acid catalysis is predicted to substantially lower the effective barrier to reaction.

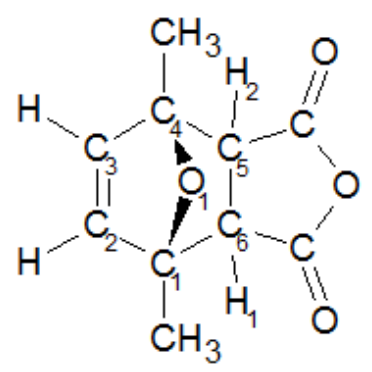

Figure 1. Atom labels. 
(a)

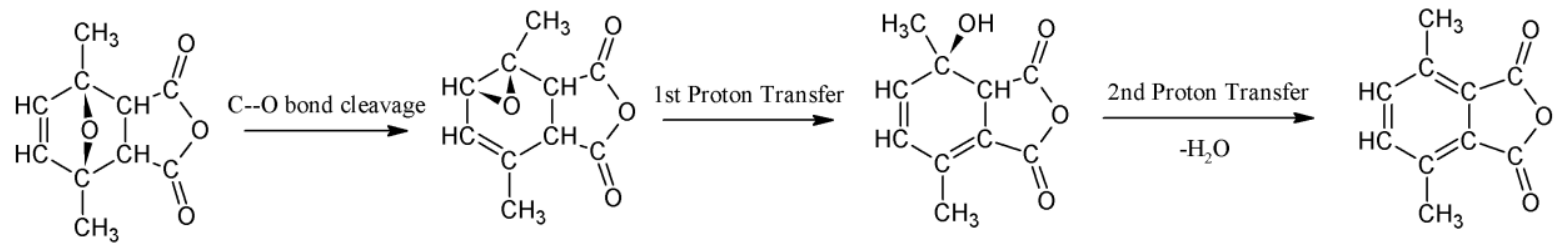

(b)

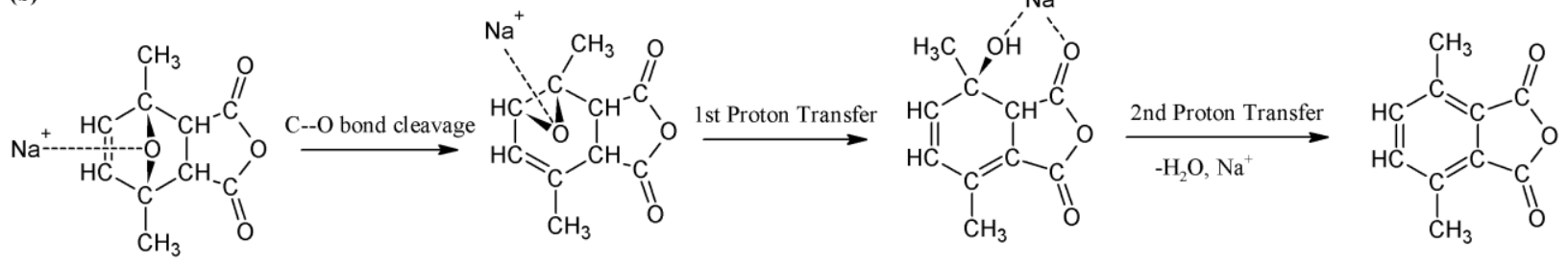

(c)

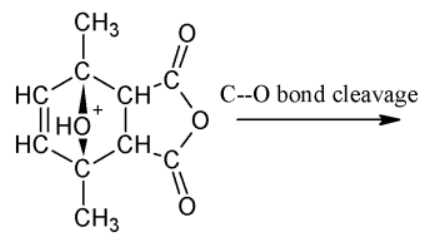<smiles>C[C+]1C=CC2(C)OC1C1C(=O)OC(=O)C12</smiles>

1st Proton Transfer $-\mathrm{H}_{2} \mathrm{O}$<smiles>CC1=C2C(=O)OC(=O)C2[C+](C)C=C1</smiles>

Figure 2. Cycloadduct dehydration mechanisms: (a) Uncatalyzed; (b) Lewis acid catalyzed; and (c) Brønsted acid catalyzed. 


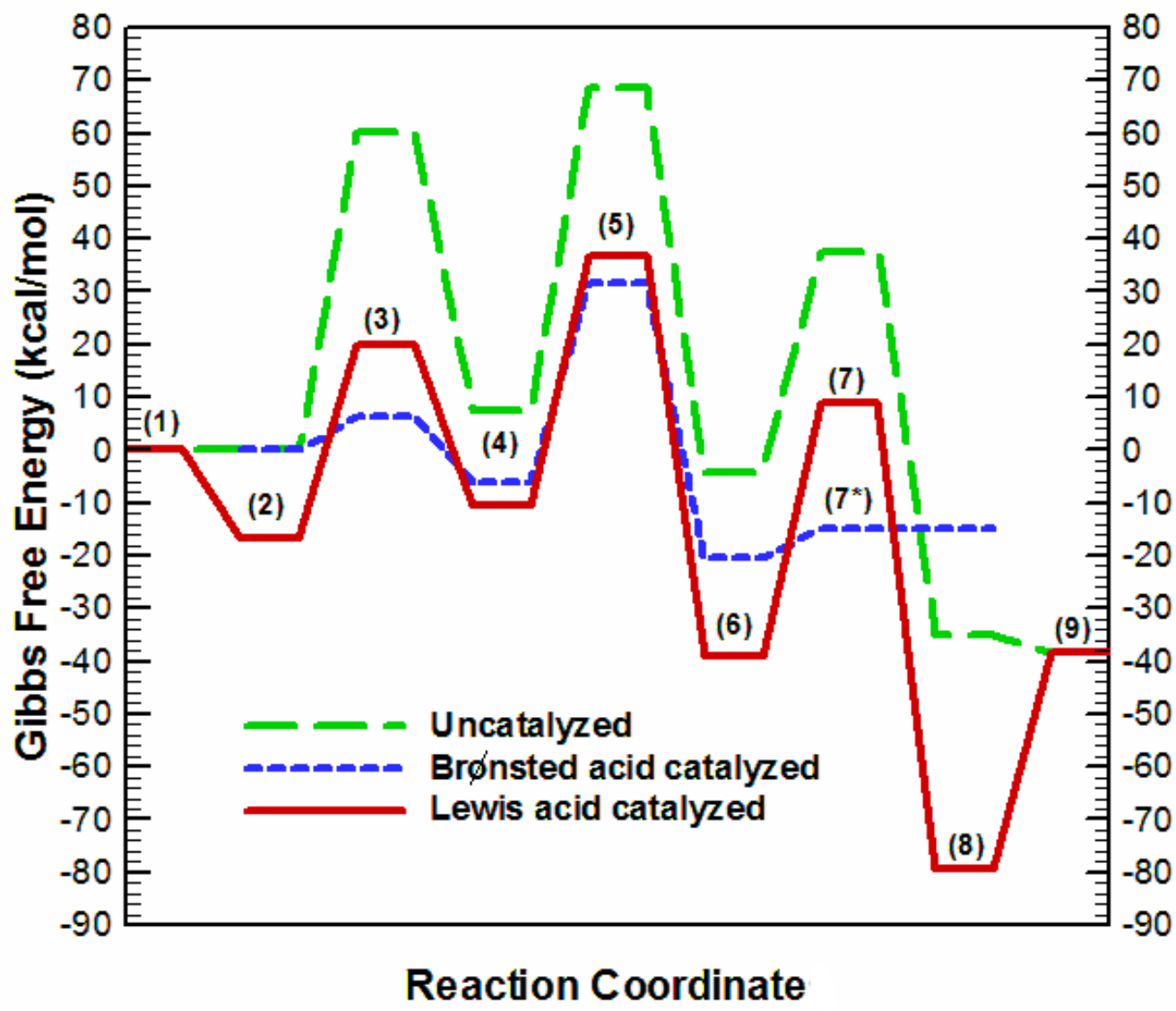

Figure 3. Gibbs free energy profiles of dehydration reaction in vacuum, uncatalyzed, and with a Lewis $\left(\mathrm{Na}^{+}\right)$or Brønsted acid $\left(\mathrm{H}^{+}\right)$catalyst. Levels are indexed as following: (1) Separated reactants (except Brønsted acid case) (2) Cycloadduct complexed with catalyst (Lewis and Brønsted catalyzed cases) (3) Transition state for C-O bond cleavage (4) Intermediate after breaking the $\mathrm{C}-\mathrm{O}$ bond (5) Transition state for the first intramolecular proton transfer (6) Intermediate after the first proton transfer (7) Transition state for the second intramolecular proton transfer and elimination of water (uncatalyzed and Lewis acid cases) $\left(7^{*}\right)$ Water molecule separated from the protonated aromatic product (Brønsted acid 
case) (8) Aromatic product coordinated to catalyst with weakly bound water molecule (9) Water molecule, catalyst and aromatic product infinitely separated.

Our results can be compared to those of Nikbin and co-workers ${ }^{11}$, who examined the cycloaddition of DMF and ethylene to form another oxa-norbornene derivative, followed by dehydration to p-xylene. Their cycloadduct has only a single six-member ring and no anhydride functionality. The mechanisms for the uncatalyzed and catalyzed dehydrations are similar to those described here, but the barrier found here for the first proton transfer step is higher in all cases. This is not a matter of bond strength, as the $\mathrm{C}_{6}-\mathrm{H}_{1}$ bond is weaker in our intermediate, by about $1 \mathrm{kcal} / \mathrm{mol}$. Instead, the higher barriers to proton transfer reflect the geometric constraints imposed by the five-member anhydride ring. For example, in the uncatalyzed case, the $\mathrm{O}_{1}-\mathrm{H}_{1}$ distance in the intermediate leading to the first proton transfer (Fig. 2) is about $0.3 \AA$ longer in our case than in the case of Nikbin et al. The higher barriers to proton transfer are a result of the greater distortion needed to reach the transition state. Nikbin et al. modeled the Brønsted acid catalyst as a proton, just as in the present work. Experimental work using a Brønsted acidic zeolite are consistent with model predictions that this mechanism is effective for dehydration. ${ }^{36}$

\subsection{Solvent effect}

\subsubsection{Uncatalyzed}


Table 3 shows the Gibbs free energies of transition states and intermediates along the reaction path for uncatalyzed dehydration in a broad range of solvents. Free energies are given with reference to the cycloadduct in each solvent. The free energies of all the transiton states decrease by $1-5 \mathrm{kcal} / \mathrm{mol}$ with solvent dielectric constant, with the strongest effect observed on the first transition state. The second step is rate limiting in all cases. None of the solvents reduce the activation barrier enough to make the uncatalyzed reaction practical.

Table 3. Gibbs Free energy levels of uncatalyzed dehydration reaction in vacuum and several solvents, in units of kcal/mol. States are indexed as in Fig. 3. Solvent dielectric constants $(\varepsilon)$ are listed in the first row.

\begin{tabular}{|c|c|c|c|c|c|c|c|}
\hline Index & Vacuum & Heptane & Diethylether & THF & Acetone & Acetonitrile & DMSO \\
\hline$\epsilon$ & 1.0 & 1.9 & 4.2 & 7.4 & 20.5 & 35.7 & 46.8 \\
\hline 1 & 0.0 & 0.0 & 0.0 & 0.0 & 0.0 & 0.0 & 0.0 \\
\hline 3 & 60.2 & 58.3 & 56.5 & 55.7 & 54.2 & 54.0 & 54.5 \\
\hline 4 & 7.6 & 7.1 & 7.6 & 7.6 & 7.5 & 7.5 & 7.3 \\
\hline 5 & 68.7 & 67.4 & 66.6 & 66.2 & 65.5 & 65.6 & 65.8 \\
\hline 6 & -4.2 & -4.7 & -4.8 & -5.1 & -5.8 & -6.2 & -5.4 \\
\hline 7 & 37.6 & 36.6 & 36.0 & 35.7 & 35.2 & 34.9 & 35.6 \\
\hline 8 & -35.2 & -34.1 & -34.9 & -34.5 & -35.3 & -35.2 & -34.8 \\
\hline 9 & -38.3 & -41.2 & -40.9 & -40.9 & -41.5 & -41.5 & -40.9 \\
\hline
\end{tabular}

\subsubsection{Lewis acid catalysis}

Table 4 shows the free energies of transition states and intermediates in the Lewis acid catalyzed dehydration with $\mathrm{Na}^{+}$as the catalyst, for a number of solvents. Two specific cases, at low and high dielectric constants, are illustrated in Fig. 4. In the reference state, the cycloadduct and the ion are separately solvated, as in state 1 of Fig. 3. The energetic span is also reported in Table 4, 
and there is a strong solvent effect, which reduces the energetic span by as much as $19 \mathrm{kcal} / \mathrm{mol}$. This is due to differential solvation of the turnover-determining transition state (TDTS, state 5) and the turnover-determining intermediate (TDI, state 8). Both states are destabilized in solvent, but to different degrees (Fig. 4). Table 4 includes energies for state $8^{*}$, in which the water molecule is removed from the complex of the catalyst and aromatic product, showing that this state does not increase the energetic span. In all solvents except heptane, the energetic span is lower than the activation barrier in the uncatalyzed case. This illustrates the significant role that solvent can play in the kinetics of these reactions. However, in this case the energetic span remains large enough that very high temperatures would be required to achieve useful turnover frequencies.

Table 4. Gibbs free energy profiles of Lewis acid $\left(\mathrm{Na}^{+}\right)$catalyzed dehydration reaction in vacuum and several solvents, along with the energetic span, $\delta E$, in $\mathrm{kcal} / \mathrm{mol}$. States are indexed as in Fig. 3, with the addition of $8^{*}$, the state where water is separated from the complex of the aromatic product with the catalyst. Solvent dielectric constants $(\varepsilon)$ are listed in the first row.

\begin{tabular}{|c|c|c|c|c|c|c|c|}
\hline Index & Vacuum & Heptane & Diethylether & THF & Acetone & Acetonitrile & DMSO \\
\hline $\boldsymbol{\epsilon}$ & 1.0 & 1.9 & 4.2 & 7.4 & 20.5 & 35.7 & 46.8 \\
\hline 1 & 0.0 & 0.0 & 0.0 & 0.0 & 0.0 & 0.0 & 0.0 \\
\hline 2 & -16.6 & -8.2 & -5.4 & -4.9 & -4.5 & -4.6 & -4.7 \\
\hline 3 & 19.8 & 29.7 & 35.4 & 36.6 & 37.2 & 37.0 & 37.5 \\
\hline 4 & -10.3 & -1.8 & 1.8 & 2.0 & 2.6 & 2.8 & 2.9 \\
\hline 5 & 36.9 & 46.4 & 51.6 & 52.5 & 53.1 & 52.9 & 53.4 \\
\hline 6 & -38.9 & -27.6 & -19.9 & -18.1 & -17.4 & -17.8 & -16.1 \\
\hline 7 & 9.0 & 21.3 & 28.4 & 29.8 & 31.0 & 30.2 & 32.1 \\
\hline 8 & -79.3 & -64.3 & -54.4 & -50.9 & -48.4 & -47.8 & -46.7 \\
\hline $8 *$ & -67.1 & -56.5 & -49.0 & -47.6 & -44.9 & -45.2 & -43.9 \\
\hline
\end{tabular}




\begin{tabular}{|c|c|c|c|c|c|c|c|}
\hline 9 & -38.3 & -41.2 & -40.9 & -40.9 & -41.5 & -41.5 & -40.9 \\
\hline$\delta E$ & $\mathbf{7 7 . 9}$ & $\mathbf{6 9 . 6}$ & $\mathbf{6 5 . 1}$ & $\mathbf{6 2 . 5}$ & $\mathbf{6 0 . 1}$ & $\mathbf{5 9 . 2}$ & $\mathbf{5 9 . 1}$ \\
\hline
\end{tabular}

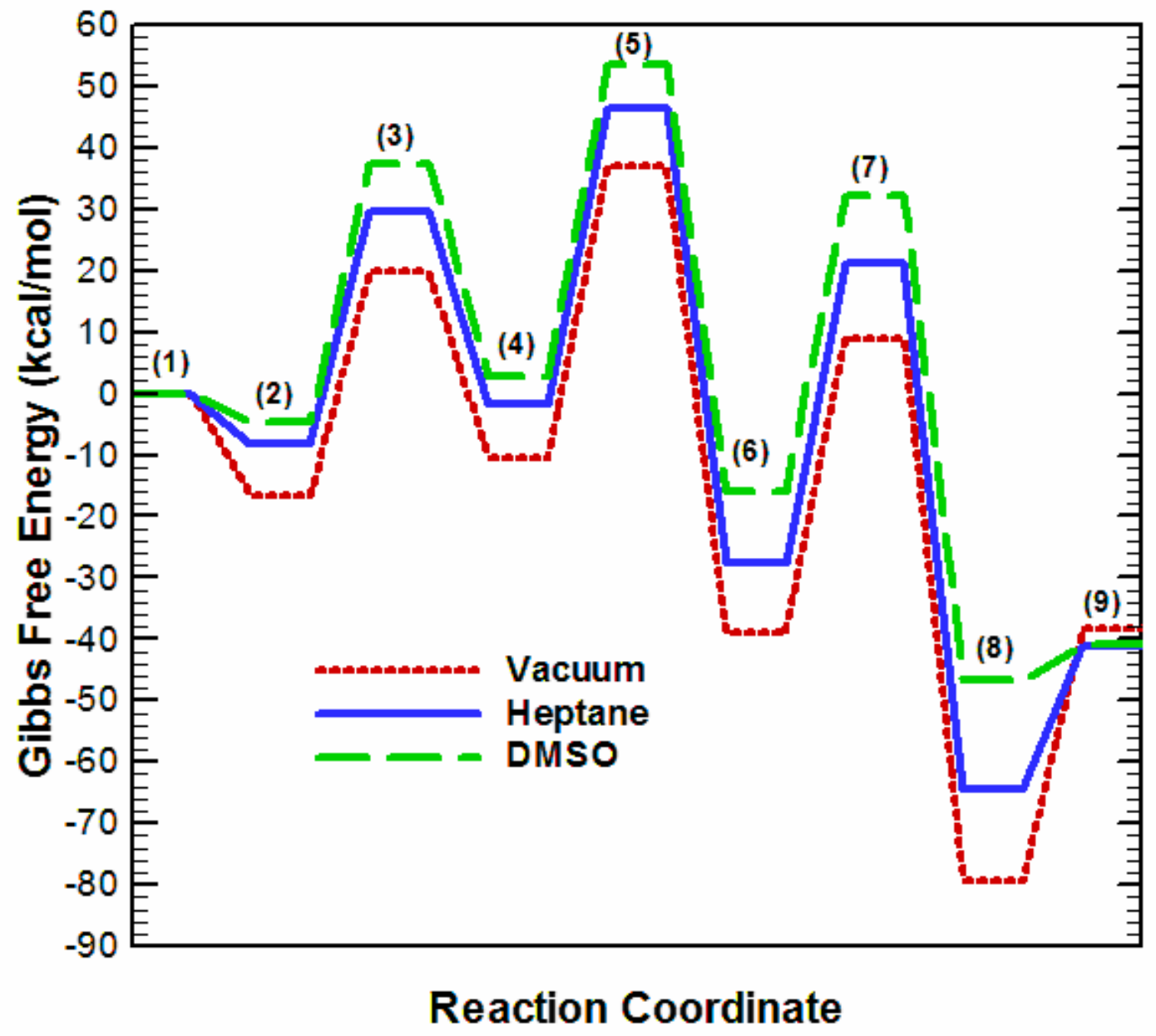

Figure 4. Free energy profiles for Lewis acid catalyzed dehydration in two representative solvents, with vacuum profile. States are indexed as in Fig. 3.

3.2.3. Brønsted acid catalysis 
Table 5 shows the free energy profile for Brønsted acid catalyzed dehydration in vacuum and several solvents, along with the overall free energy of reaction and the energetic span. Profiles for two specific solvents, with low and high dielectric constants, are depicted in Fig. 5. The reference energy corresponds to the protonated cycloadduct in the solvent; all other species shown are protonated. As in the gas phase, this information, along with the overall reaction energy, is enough to calculate the energetic span. Thus, there is no need to estimate the energy of protonation or deprotonation processes, or the solvation energy of the proton.

As shown in Table 5, the energetic span in solvent is even smaller than in the gas phase. The transition state energies and the energetic span show only small variations with solvent. The largest change observed is in the stability of state 6, with a water molecule interacting weakly with the protonated aromatic product. In vacuum and heptane, this state has lower free energy than state $7^{*}$, where the water is completely separated. For more polar solvents, state 6 has higher free energy than state $7 *$, and water spontaneously dissociates from the complex. This means that the TDI shifts from state 6 for vacuum and heptane to state $7^{*}$ for the other cases. In all cases, the energetic span is small enough for dehydration by this mechanism to occur readily at room temperature.

Table 5. Gibbs free energy levels of Brønsted acid catalyzed dehydration reaction in vacuum and several solvents, in units of $\mathrm{kcal} / \mathrm{mol}$. The free energy of reaction and the energetic span are included. States are indexed as in Fig. 3. Solvent dielectric constants $(\varepsilon)$ are listed in the first row.

\begin{tabular}{|c|c|c|c|c|c|c|c|}
\hline Index & Vacuum & Heptane & Diethylether & THF & Acetone & Acetonitrile & DMSO \\
\hline$\epsilon$ & 1 & 1.9 & 4.2 & 7.4 & 20.5 & 35.7 & 46.8 \\
\hline 2 & 0.0 & 0.0 & 0.0 & 0.0 & 0.0 & 0.0 & 0.0 \\
\hline 3 & 6.3 & 5.5 & 7.5 & 7.6 & 7.3 & 8.1 & 7.6 \\
\hline
\end{tabular}




\begin{tabular}{|c|c|c|c|c|c|c|c|}
\hline 4 & -6.2 & -5.0 & -4.3 & -3.9 & -3.5 & -2.7 & -3.6 \\
\hline 5 & 31.6 & 32.1 & 33.2 & 33.3 & 33.4 & 33.7 & 33.3 \\
\hline 6 & -20.6 & -17.7 & -14.6 & -12.7 & -12.5 & -11.3 & -11.7 \\
\hline $7^{*}$ & -14.8 & -16.5 & -16.2 & -16.7 & -17.1 & -16.0 & -17.3 \\
\hline$\Delta G_{r}$ & -38.3 & -41.2 & -40.9 & -40.9 & -41.5 & -41.5 & -40.9 \\
\hline$\delta E$ & $\mathbf{1 3 . 8}$ & $\mathbf{8 . 5}$ & $\mathbf{8 . 5}$ & $\mathbf{9 . 1}$ & $\mathbf{9 . 0}$ & $\mathbf{8 . 1}$ & $\mathbf{9 . 6}$ \\
\hline
\end{tabular}

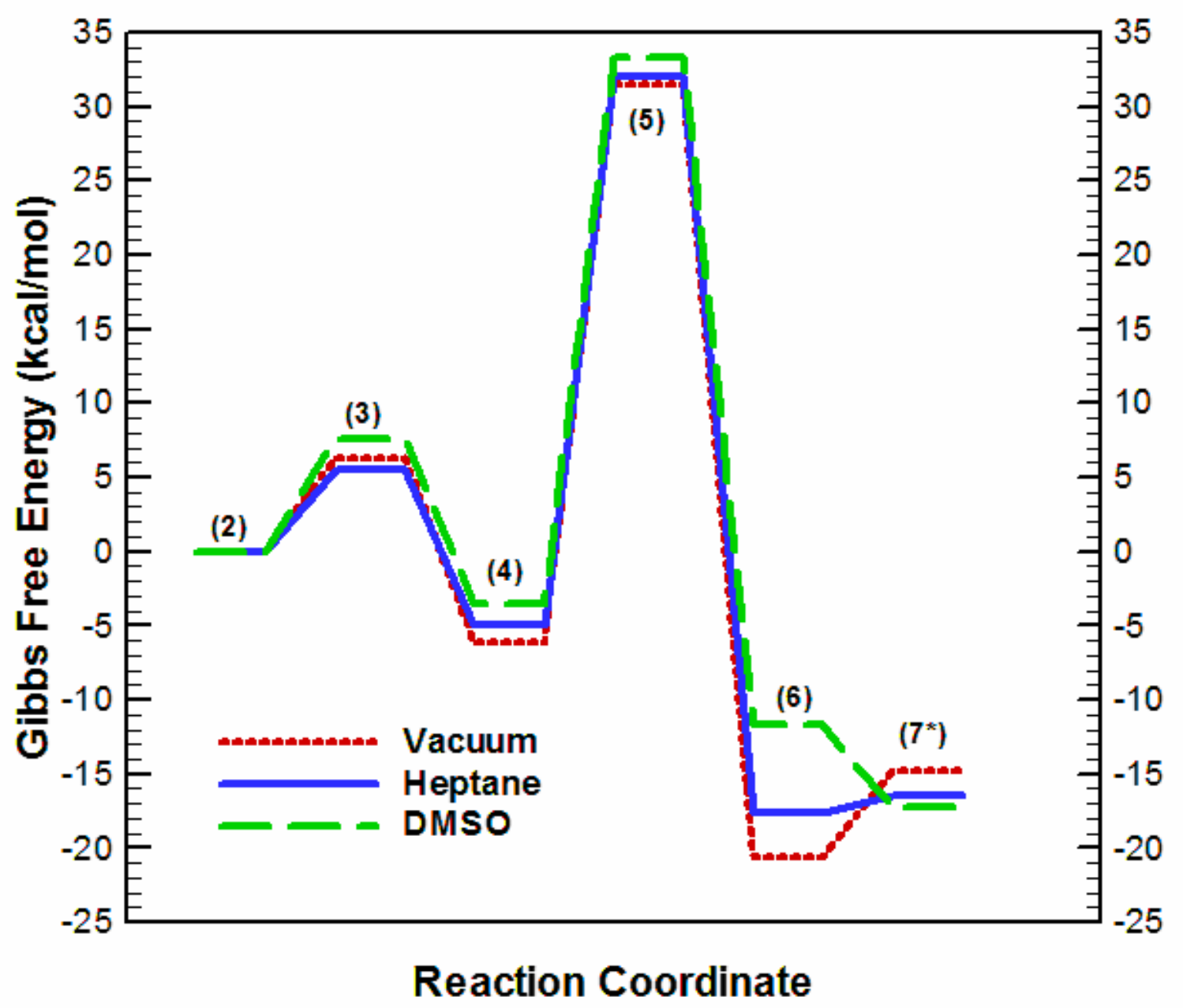

Figure 5. Free energy profiles for Brønsted acid catalyzed dehydration in two representative solvents, with vacuum profile. States are indexed as in Fig. 3. 


\section{Conclusion}

We have used density functional theory to investigate the dehydration of the Diels-Alder cycloaddition product of DMF and MA to form 3,6-dimethyl phthalic anhydride. We find that the uncatalyzed dehydration has very high activation barriers, and is not thermally feasible. Lewis acid catalysis requires even higher temperatures in the vacuum. Solvents can substantially reduce the energetic span for Lewis acid catalyzed dehydration, but very high temperatures would still be needed. For the Brønsted acid case, the energetic span is low enough to allow reaction at room temperature, and this appears to be the most promising strategy for dehydration. Solvent reduces the energetic span even further for Brønsted acid catalysis.

Our previous work showed that the Diels-Alder cycloaddition of DMF and MA can be catalyzed by either a Lewis or Brønsted acid. ${ }^{17}$ Such Brønsted acid catalysis for the cycloaddition is rare in studies of biomass conversion, and occurs with MA as dienophile, but not ethylene, for example. The present results suggest that a single catalyst could be effective for both steps in the conversion of DMF and MA to 3,6-dimethyl phthalic anhydride. These results provide a baseline for future more realistic studies with zeolite structure and explicit solvent molecules.

\section{AUTHOR INFORMATION}

\section{Corresponding Author}

* Taha Salavati-fard, Email: taha@udel.edu, Tel: +1 3028316381.

\section{Author Contributions}


The manuscript was written through contributions of all authors. All authors have given approval to the final version of the manuscript.

\section{Acknowledgement}

This material is based upon work supported as part of the Catalysis Center for Energy Innovation, an Energy Frontier Research Center funded by the U.S. Department of Energy, Office of Science, Office of Basic Energy Sciences under Award Number DE-SC0001004. This research used resources of the National Energy Research Scientific Computing Center, a DOE Office of Science User Facility supported by the Office of Science of the U.S. Department of Energy under Contract No. DE-AC02-05CH11231.

\section{References}

[1] Gallezot, P. Conversion of biomass to selected chemical products. Chem. Soc. Rev. 2012, 41, 1538-1558.

[2] Christensen, C. H.; Rass-Hansen, J.; Marsden, C. C,; Taarning, E,; Egeblad, K. The renewable chemicals industry. ChemSusChem 2008, 1, 283-289.

[3] Chheda, J. N.; Huber, G. W.; Dumesic, J. A. Liquid-phase catalytic processing of biomass-derived oxygenated hydrocarbons to fuels and chemicals. Angew. Chem. Int. Ed. 2007, 46, 7164-7183.

[4] Román-Leshkov, Y.; Barrett, C. J.; Liu, Z. Y.; Dumesic, J. A. Production of dimethylfuran for liquid fuels from biomass-derived carbohydrates. Nature 2007, 447, 982-985. 
[5] Huber, G. W.; Chheda, J. N.; Barrett, C. J.; Dumesic, J. A. Production of liquid alkanes by aqueous-phase processing of biomass-derived carbohydrates. Science 2005, 308, 1446-1450.

[6] Cortright, R. D.; Davda, R. R.; Dumesic, J. A. Hydrogen from catalytic reforming of biomass-derived hydrocarbons in liquid water. Nature 2002, 418, 964-967.

[7] Brion, F. On the lewis acid catalyzed diels-alder reaction of furan. regio-and stereospecific synthesis of substituted cyclohexenols and cyclohexadienols. Tetrahedron Lett. 1982, 23, 52995302.

[8] Nishimura, S.; Ikeda, N.; Ebitani, K. Selective hydrogenation of biomass-derived 5hydroxymethylfurfural (HMF) to 2, 5-dimethylfuran (DMF) under atmospheric hydrogen pressure over carbon supported PdAu bimetallic catalyst. Catal. Today 2014, 232, 89-98.

[9] Huang, Y. B.; Chen, M. Y.; Yan, L.; Guo, Q. X.; Fu, Y. Nickel-tungsten carbide catalysts for the production of 2, 5-dimethylfuran from biomass-derived molecules. ChemSusChem 2014, 7, 1068-1072.

[10] Mahmoud, E.; Watson, D. A.; Lobo, R. F. Renewable production of phthalic anhydride from biomass-derived furan and maleic anhydride. Green Chem. 2014, 16, 167-175.

[11] Nikbin, N.; Do, P. T.; Caratzoulas, S.; Lobo, R. F.; Dauenhauer, P. J.; Vlachos, D. G. A DFT study of the acid-catalyzed conversion of 2,5-dimethylfuran and ethylene to p-xylene. $J$. Catal. 2013, 297, 35-43.

[12] Nikbin, N.; Feng, S.; Caratzoulas, S.; Vlachos, D. G. P-xylene formation by dehydrative aromatization of a Diels-Alder product in Lewis and Brønsted acidic zeolites. J. Phys. Chem. C $2014,118,24415-24424$. 
[13] Mellmer, M. A.; Sener, C.; Gallo, J. M.; Luterbacher, J. S.; Alonso, D. M.; Dumesic, J. A. Solvent effects in acid-catalyzed biomass conversion reactions. Angew. Chem. Int. Ed. 2014, 53, $11872-11875$.

[14] Mellmer, M. A.; Alonso, D. M.; Luterbacher, J. S.; Gallo, J. M.; Dumesic, J. A. Effects of $\gamma$ valerolactone in hydrolysis of lignocellulosic biomass to monosaccharides. Green Chem. 2014, $16,4659-4662$.

[15] Liu, Z.; Zhang, F. S. Effects of various solvents on the liquefaction of biomass to produce fuels and chemical feedstocks. Energy Convers. Manage. 2008, 49, 3498-3504.

[16] Kruse, A.; Dahmen, N. Water-A magic solvent for biomass conversion. J. Supercrit. Fluids 2015, 96, 36-45.

[17] Salavati-fard, T.; Caratzoulas, S.; Doren, D. J. DFT study of solvent effects in acidcatalyzed Diels-Alder cycloadditions of 2, 5-dimethylfuran and maleic anhydride. J. Phys. Chem. A 2015, 119, 9834-9843.

[18] Frisch, M. J.; Trucks, G. W.; Schlegel, H. B.; Scuseria, G. E.; Robb, M. A.; Cheeseman, J. R.; Scalmani, G.; Barone, V.; Mennucci, B.; Petersson, G. A., et al. Gaussian 09, revision A.2; Gaussian Inc.: Wallingford, CT, 2009.

[19] Zhao, Y.; Truhlar, D. G. Density functionals with broad applicability in chemistry. Acc. Chem. Res. 2008, 41, 157-167.

[20] Zhao, Y.; Truhlar, D. G. The m06 suite of density functionals for main group thermochemistry, thermochemical kinetics, noncovalent interactions, excited states, and transition elements: two new functionals and systematic testing of four m06-class functionals and 12 other functionals. Theor. Chem. Acc. 2008, 120, 215-241. 
[21] Marenich, A. V.; Cramer, C. J.; Truhlar, D. G. Universal solvation model based on solute electron density and on a continuum model of the solvent defined by the bulk dielectric constant and atomic surface tensions. J. Phys. Chem. B 2009, 113, 6378-6396.

[22] Reed, A. E.; Weinstock, R. B.; Weinhold, F. Natural population analysis. J. Chem. Phys. $1985,83,735-746$.

[23] Meister, J.; Schwarz, W. H. E. Principal components of ionicity. J. Phys. Chem. 1994, 98, $8245-8252$.

[24] Glendening, E. D.; Reed, A. E.; Carpenter, J. E.; Weinhold F. NBO Version 3.1.

[25] Wannakao, S.; Warakulwit, C.; Kongpatpanich, K.; Probst, M.; Limtrakul, J. Methane activation in gold cation-exchanged zeolites: A DFT study. ACS Catal. 2012, 2, 986-992.

[26] Chowdhuri, S.; Chandra, A. Molecular dynamics simulations of aqueous $\mathrm{NaCl}$ and $\mathrm{KCl}$ solutions: Effects of ion concentration on the single-particle, pair, and collective dynamical properties of ions and water molecules. J. Chem. Phys. 2001, 115, 3732-3741.

[27] Bader R. F. W. Atoms in molecules. John Wiley \& Sons, Ltd; 1990.

[28] Bader R. F. W. A quantum theory of molecular structure and its applications. Chem. Rev. 1991, 91, 893-928.

[29] Bader R. F. W. A bond path: a universal indicator of bonded interactions. J. Phys. Chem. A $1998,102,7314-7323$.

[30] Popelier P. L. On the full topology of the Laplacian of the electron density. Coord. Chem. Rev. 2000, 197, 169-189. 
[31] Gibbs G. V.; Hill F. C.; Boisen M. B.; Downs R. T. Power law relationships between bond length, bond strength and electron density distributions. Phys. Chem. Miner. 1998, 25, 585-590.

[32] Kozuch S.; Shaik S. How to conceptualize catalytic cycles? The energetic span model. Acc. Chem. Res. 2010, 44, 101-110.

[33] Kozuch, S. A refinement of everyday thinking: the energetic span model for kinetic assessment of catalytic cycles. WIREs Comput. Mol. Sci. 2012, 2, 795-815.

[34] Kozuch, S.; Martin J. M. "Turning over" definitions in catalytic cycles. ACS Catal. 2012, 2, 2787-2794.

[35] Kozuch, S.; Shaik, S. A combined kinetic-quantum mechanical model for assessment of catalytic cycles: application to cross-coupling and heck reactions. J. Am. Chem. Soc. 2006, 128, $3355-3365$.

[36] Williams C. L.; Chang C. C.; Do P.; Nikbin N.; Caratzoulas S.; Vlachos D. G.; Lobo R. F.; Fan W.; Dauenhauer P. J. Cycloaddition of biomass-derived furans for catalytic production of renewable p-xylene. ACS Catal. 2012, 2, 935-939. 


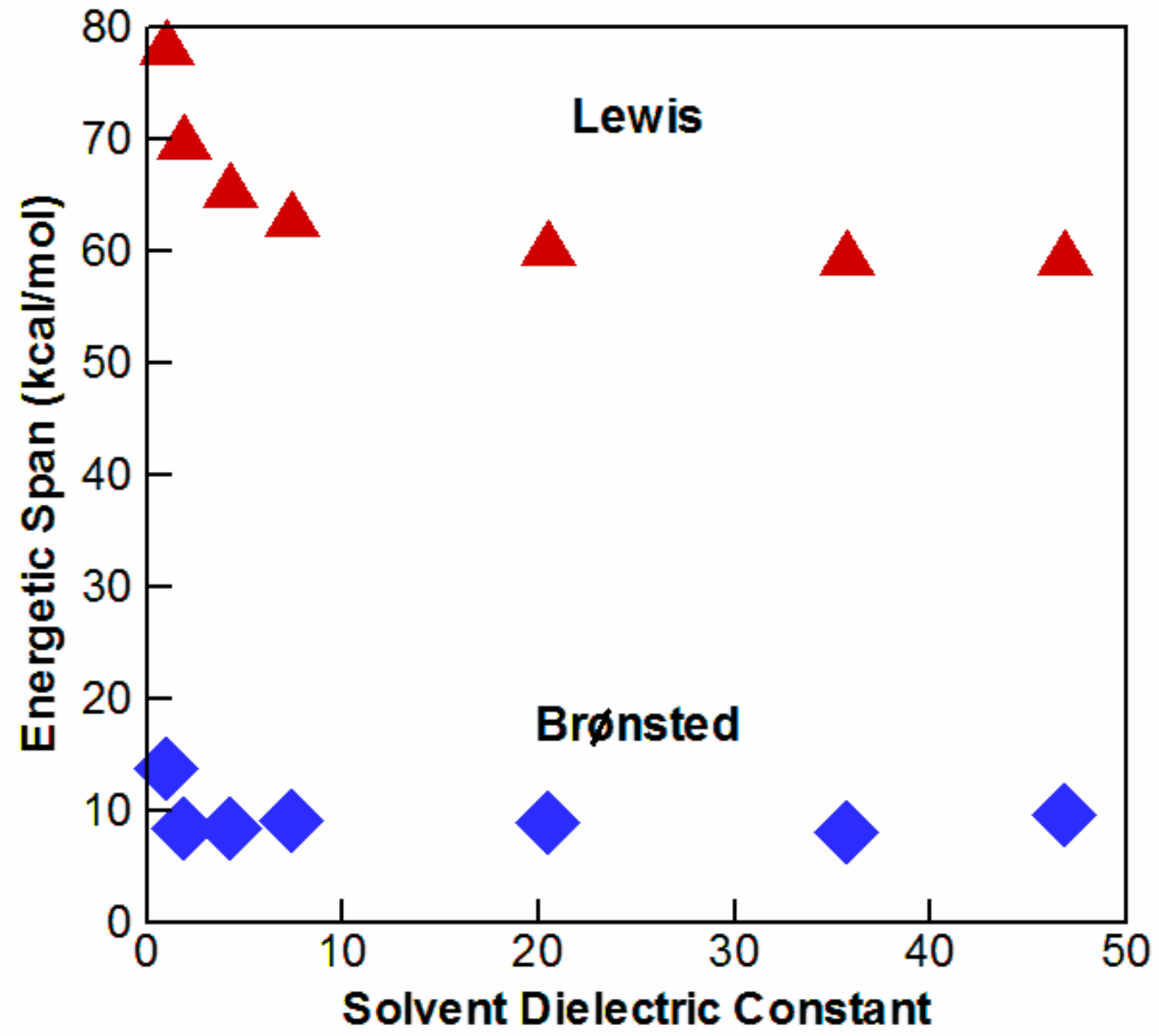

